\title{
Progressive Multifocal Leukoencephalopathy in an Immunocompetent Patient?
}

\author{
Krisztina K. Johansen ${ }^{a, d} \quad$ Sverre H. Torp ${ }^{b, e} \quad$ Jana Rydland ${ }^{c}$ \\ Jan O. Aasly ${ }^{a, d}$ \\ Departments of ${ }^{\mathrm{a}}$ Neurology, ${ }^{\mathrm{b}}$ Pathology and ${ }^{\mathrm{C}}$ Radiology, St Olavs University Hospital and \\ Departments of ${ }^{\mathrm{d}}$ Neuroscience and 'Laboratory Medicine, Children's and Women's \\ Health, Norwegian University of Science and Technology, Trondheim, Norway
}

\section{Key Words}

Hypogammaglobulinemia · Idiopathic CD4+ T-lymphocytopenia · JC virus · Progressive multifocal leukoencephalopathy

\begin{abstract}
Background: Progressive multifocal leukoencephalopathy (PML) is a rapidly progressive, potentially fatal, demyelinating disease affecting immunosuppressed patients. PML is rarely reported in cases with no underlying disease or immunosuppression-associated condition. Case Report: We present a 72-year-old previously healthy woman who developed a progressive neurological condition affecting the entire nervous system which led to her death within 5 months. PML was diagnosed at autopsy. Conclusion: PML should be considered in patients with progressive neurological disorders involving the white matter, even in the absence of previous immunomodulatory treatment or immunosuppression.
\end{abstract}

(c) 2013 S. Karger AG, Basel

\section{Introduction}

Progressive multifocal encephalopathy (PML) is a potentially fatal, demyelinating disease of the central nervous system caused by a papillomavirus called JC virus [1]. This entity presents with a wide clinical spectrum and is pathologically characterized by multiple demyelinating lesions, oligodendrocytes with enlarged nuclei, inclusions and bizarre astrocytes. A possible diagnosis is based on clinical manifestations, imaging findings and JC-

Krisztina K. Johansen

Department of Neurology, St Olavs University Hospital

E. Griegs gt, 8

NO-7006 Trondheim (Norway)

E-Mail krisztina.k.johansen@ntnu.no 
Johansen et al.: Progressive Multifocal Leukoencephalopathy in an Immunocompetent Patient?

virus-positive PCR in the cerebrospinal fluid (CSF), but the definite diagnosis is made by biopsy or at autopsy.

JC virus is widespread, reportedly infecting up to $75 \%$ of the general population. It is mostly asymptomatic. The exact pathogenesis is still unclear, but an initial infection without apparent illness is assumed to occur in adolescence. The virus might become latent and then, under certain immunosuppressed conditions, reactivate and cause PML [2]. PML mainly occurs as a complication in hematological malignancies, in lymphoproliferative disorders, in immunosuppressed patients and most commonly in patients with AIDS. The increasing use of various immunosuppressive and immunomodulatory agents has led to a higher incidence of PML. Numerous reports have been published on the association between PML and the use of monoclonal antibodies, such as efalizumab, natalizumab and rituximab in patients with psoriasis, Crohn's disease and multiple sclerosis [3]. Autoimmune disorders with various chronic organ failures can be complicated by this fatal demyelinating disease, but it is rarely seen in immunocompetent subjects without underlying disease [4].

We present a case of PML with no underlying disease where the diagnosis was made at autopsy.

\section{Case Presentation}

A 72-year-old female was admitted to her local hospital because of right-sided hemiparesis and dysphasia. She had a history of well-regulated hypertension and total alopecia at age 40 years with spontaneous improvement. She had no known risk factors for immunosuppression. Her mother had suffered a stroke at the age of 70 years and no other family history of disease was reported. Three months prior to admission, the patient noted fluctuating small, red, itchy skin eruptions without fever or other accompanying infection signs. CT of the brain showed a hypodense lesion in the left parietal region, considered to be of vascular genesis. Acetylsalicylic acid and simvastatin were added to her antihypertensive medication.

One month later, a gradual worsening occurred. Three months after the initial neurological signs, a new CT scan revealed an enlarged hypodense lesion in the left parietal region and a minor lesion in the right dorsal parietal region. The patient's condition deteriorated considerably with increasing neurological symptoms, personality changes, aphasia, apraxia and right-sided hemiplegia.

Laboratory tests showed a normal hemoglobin level and white blood cell and neutrophil counts. The lymphocyte count was low (12-15\%; normal range 21-43) with low levels of serum IgG (4.7 g/l; normal range 6.1-14.9) and IgM (0.2 g/l; normal range 0.4-2.1), but a normal IgA level and no monoclonal component were seen. C-reactive protein, SR level, antinuclear antibodies, antineutrophil cytoplasmic antibody, double-stranded DNA, cardiolipin antibodies and rheumatoid factors were normal. No kidney, liver or thyroid abnormalities were observed. Cancer marker tests were normal. Serological tests for Epstein-Barr virus, cytomegalovirus, borrelia and syphilis were all negative. An HIV test was not performed. CSF analysis revealed a normal cell count and normal protein and glucose levels; there was no sign of intrathecal immunoglobulin synthesis. PCR for neurotropic viruses (herpes simplex virus, varicella zoster virus, cytomegalovirus, Epstein-Barr virus and enterovirus) and borrelia were negative. The electroencephalogram showed bilateral theta and delta waves, but no epileptic activity.

A whole-body CT scan revealed bilateral kidney cysts, but no sign of malignancy or other pathological findings. MRI of the brain showed widespread white-matter lesions in both 
Johansen et al.: Progressive Multifocal Leukoencephalopathy in an Immunocompetent Patient?

hemispheres, more extensively on the left side involving the frontal, parietal and occipital lobes (fig. 1). The lesions were localized in the subcortical white matter. Examination revealed several small foci on contrast-enhancement in the right parietal lobe. Restricted diffusion was not noted. Magnetic resonance spectroscopy performed with multivoxal chemical shift technique over the right frontoparietal lobe showed unspecific changes with an elevated choline-to-creatine ratio and reduced $\mathrm{N}$-acetylaspartate levels.

The patient deteriorated and the planned stereotactic brain biopsy was not performed because of her poor condition. She gradually progressed to a vegetative state. She passed away from the cardiopulmonary complications of bilateral bronchopneumonia 5 months after the first signs of disease.

Three days later an autopsy was performed. The bilateral bronchopneumonia, moderate coronary atherosclerosis, bilateral kidney cysts and cholecystolithiasis were confirmed. A 13-mm tumor in the ileum without atypia was detected on histopathological examination. The brain was macroscopically atrophic, most prominently in the frontal and parietal lobes. Multiple confluent demyelination foci were described in both hemispheres, but not in the brainstem or the cerebellum. The microscopic examination revealed areas with demyelination, a variable number of macrophages, enlarged purple-stained oligodendroglial nuclei and massive gliosis with gemistocytic astrocytes, some with bizarre nuclei (fig. 2). Electron microscopy showed intranuclear inclusions, and the in situ hybridization confirmed JC virus. The autopsy diagnosis was consistent with PML.

\section{Discussion}

PML occurs rarely in patients with no underlying diseases or use of immunosuppressive agents. The patient reported here only had a history of hypertension. Her initial neurological symptoms and the first CT scan led to the misdiagnosis of stroke. Gradual worsening a few months later and MRI findings were not consistent with vascular or tumor etiology. The progression of the imaging findings did not indicate a neurodegenerative disorder. Extensive blood screening did not reveal organ failure that could cause metabolic encephalopathy. Autoimmune tests for connective tissue disorders including rheumatism were all negative. A whole-body CT scan and cancer markers in the blood did not show signs of malignancy. No malignant cells were detected by cytological examination of the CSF which meant that a lymphoma of the central nervous system was not likely. As the patient did not have any underlying cause for immunosuppression and had not undergone immunosuppressive therapy, she was not tested for JC virus in the CSF. Another weakness of the testing was the missing HIV status, although an elderly, nontravelling female farmer with no blood transfusion history was not suspected of carrying the retrovirus.

As early as the 1970s, several reports were published on PML in patients with no known underlying causes and these cases were defined as primary or spontaneous PML $[5,6]$. Gheuens et al. [4] reviewed the literature of PML cases with minimal or occult immunosuppression and found 33 cases between 1966 and 2009 and 5 cases from their own series. Twenty-two of these 38 patients did not have any chronic diseases or ongoing immunosuppressive treatment, but 5 (22.7\%) were diagnosed with idiopathic CD4+ T-lymphocytopenia. In many cases, organ failure such as liver cirrhosis, kidney failure or autoimmune disorders including Sjøgren syndrome, systemic lupus erythematosis, polymyositis, dermatomyositis and sarcoidosis were mentioned in the medical histories [4, 7].

In the following years, an increasing number of publications made it more obvious that most probably both the cellular system and the humoral immune system are involved in 
PML [8]. Many chronic diseases or organ failures might be associated with a transient immunodysfunction which might promote the reactivation of the JC virus. Idiopathic CD4+ T-lymphocytopenia has been described in PML [9] and hypogammaglobulinemia can very rarely be an underlying cause [10].

A recent report on a patient treated with fumaric acid demonstrated medicationinduced lymphocytopenia and this was considered to be the most probable cause of PML [11]. These findings indicate the necessity of continuous monitoring of the immune system to avoid the reactivation of an opportunistic infection.

In many cases, PML was not suspected prior to death and diagnosis was uncovered by autopsy, as in the case in this study. A thorough work-up including an HIV test and lymphocyte subsets should have been made to uncover possible immunosuppression. Patients on immunomodulatory treatment should have regular screening of their immune system before and during therapy to decrease the risk of PML.

To date, there is no established therapy for PML. In cases associated with immunomodulatory agents like natalizumab, the withdrawal of the drug and further elimination by plasma exchange is the most important therapy for immune system recovery [12]. Various medical regimes, for instance antimalaria medication, mefloquine and the antiviral medication cidofovir, have been applied with doubtful results [13]. JC virus has been described as using serotonin receptors to infect glial cells, and mirtazapine, an HT2a receptor antagonist, has therefore been tried to inhibit this infection [14]. In a case of hypogammaglobulinemia, the use of intravenous immunoglobulin therapy has been proposed as a possible treatment, but this needs further investigation [15].

\section{Conclusion}

PML may occur in patients with no known underlying conditions associated with immunosuppression. In cases with a relatively rapid, progressive neurological disorder affecting the entire nervous system, mainly involving the white matter, it is important to consider PML to obtain an early diagnosis. Transient immunodysfunction such as idiopathic CD4+ Tlymphocytopenia or hypogammaglobulinemia might be associated with PML. The humoral and cellular immune system should be screened to detect a possible common immunodeficiency disorder that might be an underlying cause in patients who are assumed to be immunocompetent.

\section{Disclosure Statement}

The authors have no conflicts of interest to declare.

\section{References}

1 Padgett BL, Walker DL, ZuRhein GM, Eckroade RJ, Dessel BH: Cultivation of papova-like virus from human brain with progressive multifocal leucoencephalopathy. Lancet 1971;1:1257-1260.

2 White MK, Khalili K: Pathogenesis of progressive multifocal leukoencephalopathy - revisited. J Infect Dis 2011;203:578-586.

3 Koralnik IJ: Progressive multifocal leukoencephalopathy revisited: has the disease outgrown its name? Ann Neurol 2006;60:162-173.

4 Gheuens S, Pierone G, Peeters P, Koralnik IJ: Progressive multifocal leukoencephalopathy in individuals with minimal or occult immunosuppression. J Neurol Neurosurg Psychiatry 2010;81:247-254. 
Johansen et al.: Progressive Multifocal Leukoencephalopathy in an Immunocompetent Patient?

5 Bolton CF, Rozdilsky B: Primary progressive multifocal leukoencephalopathy. A case report. Neurology 1971;21:72-77.

6 Fermaglich J, Hardman JM, Earle KM: Spontaneous progressive multifocal leukoencephalopathy. Neurology 1970;20:479-484.

7 Dastmalchi M, Laki J, Lundberg IE, Iacobaeus E: Progressive multifocal leukoencephalopathy in a patient with polymyositis: case report and literature review. J Rheumatol 2012;39:1299-1303.

$>8$ Weber F, Goldmann C, Kramer M, et al: Cellular and humoral immune response in progressive multifocal leukoencephalopathy. Ann Neurol 2001;49:636-642.

-9 Haider S, Nafziger D, Gutierrez JA, Brar I, Mateo N, Fogle J: Progressive multifocal leukoencephalopathy and idiopathic CD4+ lymphocytopenia: a case report and review of reported cases. Clin Infect Dis 2000;31:E20E22.

10 Snyder MD, Storch GA, Clifford DB: Atypical PML leading to a diagnosis of common variable immunodeficiency. Neurology 2005;64:1661.

11 Ermis U, Weis J, Schulz JB: PML in a patient treated with fumaric acid. N Engl J Med 2013;368:1657-1658.

-12 Clifford DB, De Luca A, Simpson DM, Arendt G, Giovannoni G, Nath A: Natalizumab-associated progressive multifocal leukoencephalopathy in patients with multiple sclerosis: lessons from 28 cases. Lancet Neurol 2010;9:438-446.

13 Brickelmaier M, Lugovskoy A, Kartikeyan R, et al: Identification and characterization of mefloquine efficacy against JC virus in vitro. Antimicrob Agents Chemother 2009;53:1840-1849.

14 Elphick GF, Querbes W, Jordan JA, et al: The human polyomavirus, JCV, uses serotonin receptors to infect cells. Science 2004;306:1380-1383.

15 Bezrodnik L, Samara R, Krasovec S, Garcia Erro M, Sevlever G: Progressive multifocal leukoencephalopathy in a patient with hypogammaglobulinemia. Clin Infect Dis 1998;27:181-184.

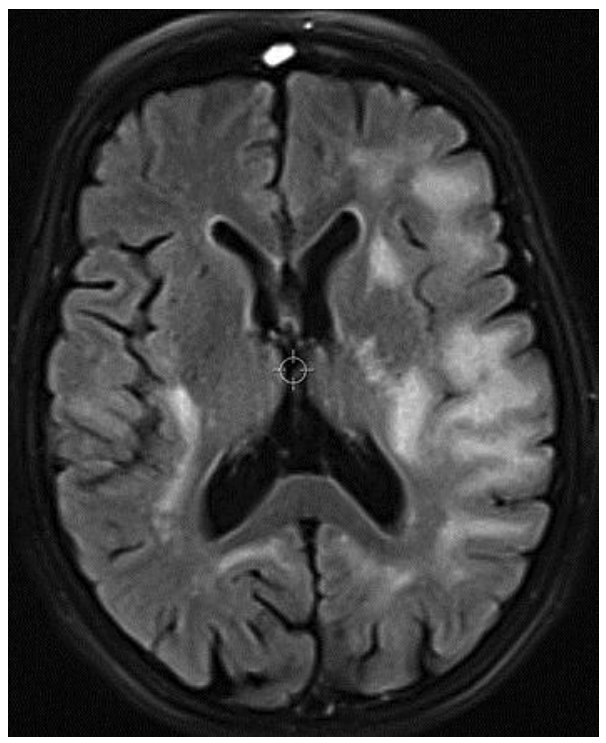

Fig. 1. Multiple patchy lesions on an axial FLAIR image located in the subcortical white matter affecting also the internal capsule on the left side. 

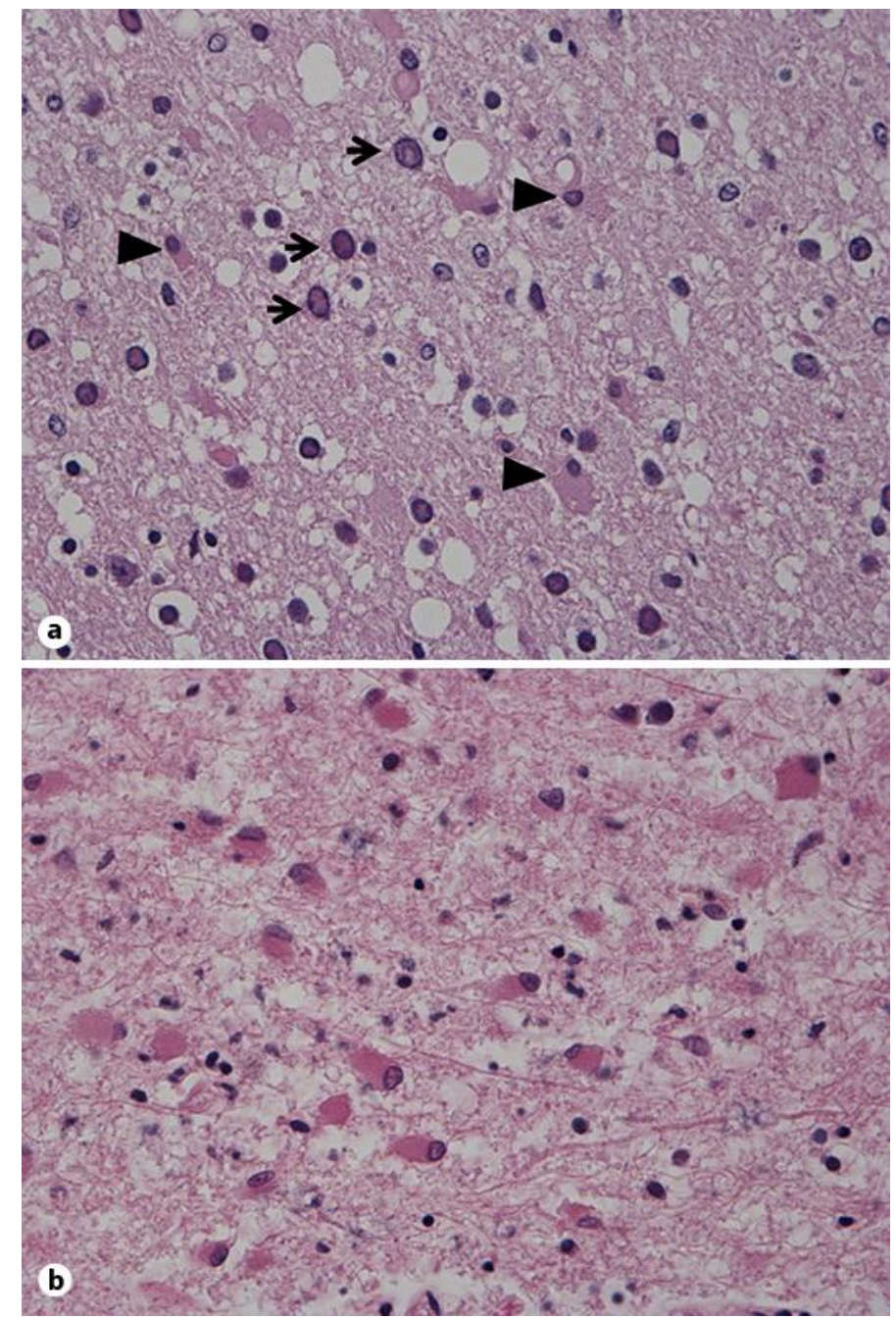

Fig. 2. a Routine HE-saffron section with enlarged abnormal oligodendroglial cell nuclei (arrow) and gliosis (arrowheads indicate gemistocytic astrocytes). $\times 40$. b Massive gliosis with gemistocytic astrocytes, some with irregular nuclei. $\times 40$. 